\title{
Further probabilistic analysis of the Fisher-Kolmogorov-Petrovskii-Piscounov equation: one sided travelling-waves
}

\author{
J.W. Harris ${ }^{\text {a }}$, S.C. Harris ${ }^{\text {a }}$, A.E. Kyprianou ${ }^{\text {b,* }}$ \\ a Department of Mathematical Sciences, University of Bath, Claverton Down, Bath, BA2 7AY, UK \\ b Mathematical Institute, Utrecht University, P.O. Box 80.010, 3508 TA, Utrecht, The Netherlands
}

Received 26 September 2004; received in revised form 3 February 2005; accepted 22 February 2005

Available online 10 October 2005

\begin{abstract}
At the heart of this article will be the study of a branching Brownian motion (BBM) with killing, where individual particles move as Brownian motions with drift $-\rho$, perform dyadic branching at rate $\beta$ and are killed on hitting the origin.

Firstly, by considering properties of the right-most particle and the extinction probability, we will provide a probabilistic proof of the classical result that the 'one-sided' FKPP travelling-wave equation of speed $-\rho$ with solutions $f:[0, \infty) \rightarrow[0,1]$ satisfying $f(0)=1$ and $f(\infty)=0$ has a unique solution with a particular asymptotic when $\rho<\sqrt{2 \beta}$, and no solutions otherwise. Our analysis is in the spirit of the standard BBM studies of [S.C. Harris, Travelling-waves for the FKPP equation via probabilistic arguments, Proc. Roy. Soc. Edinburgh Sect. A 129 (3) (1999) 503-517] and [A.E. Kyprianou, Travelling wave solutions to the K-P-P equation: alternatives to Simon Harris' probabilistic analysis, Ann. Inst. H. Poincaré Probab. Statist. 40 (1) (2004) 53-72] and includes an intuitive application of a change of measure inducing a spine decomposition that, as a by product, gives the new result that the asymptotic speed of the right-most particle in the killed BBM is $\sqrt{2 \beta}-\rho$ on the survival set.

Secondly, we introduce and discuss the convergence of an additive martingale for the killed BBM, $W_{\lambda}$, that appears of fundamental importance as well as facilitating some new results on the almost-sure exponential growth rate of the number of particles of speed $\lambda \in(0, \sqrt{2 \beta}-\rho)$.

Finally, we prove a new result for the asymptotic behaviour of the probability of finding the right-most particle with speed $\lambda>\sqrt{2 \beta}-\rho$. This result combined with Chauvin and Rouault's [B. Chauvin, A. Rouault, KPP equation and supercritical branching Brownian motion in the subcritical speed area. Application to spatial trees, Probab. Theory Related Fields 80 (2) (1988) 299-314] arguments for standard BBM readily yields an analogous Yaglom-type conditional limit theorem for the killed $\mathrm{BBM}$ and reveals $W_{\lambda}$ as the limiting Radon-Nikodým derivative when conditioning the right-most particle to travel at speed $\lambda$ into the distant future.
\end{abstract}

(c) 2005 Elsevier SAS. All rights reserved.

\footnotetext{
* Corresponding author.

E-mail addresses: j.w.harris@ bath.ac.uk (J.W. Harris), s.c.harris@bath.ac.uk (S.C. Harris), kyprianou@math.uu.nl (A.E. Kyprianou).
} 


\section{Introduction and summary of results}

A well studied non-linear PDE is the Fisher-Kolmogorov-Petrovski-Piscounov (FKPP) equation

$$
\frac{\partial u}{\partial t}=\frac{1}{2} \frac{\partial^{2} u}{\partial x^{2}}+\beta\left(u^{2}-u\right)
$$

with $u \in C^{1,2}\left(\mathbb{R}^{+} \times \mathbb{R}\right)$ and given initial condition $u(0, x):=f(x)$. The FKPP equation has been much studied by both analytic techniques, as in the original papers of Fisher [14] and Kolmogorov et al. [25], as well as probabilistic methods as found in McKean [31,32], Bramson [5,6], Uchiyama [36], Neveu [33], Chauvin and Rouault [9,10], Harris [18] and Kyprianou [27], to name just a few. In addition we refer the reader to Ikeda et al. [20-22] and Freidlin [15] for extensive discussion of the more general theory of the probabilistic representation of solutions of ordinary and partial differential equations.

Much attention has been given to FKPP solutions of the form $u(t, x)=f(x+\rho t)$ for $f \in C^{2}(\mathbb{R})$, leading to the so called FKPP travelling-wave (TW) equation

$$
\begin{aligned}
& \frac{1}{2} f^{\prime \prime}-\rho f^{\prime}+\beta\left(f^{2}-f\right)=0 \quad \text { on } \mathbb{R} \\
& f(-\infty)=1, \\
& f(\infty)=0
\end{aligned}
$$

with wave speed $\rho$. It is well known that monotone travelling-waves exist and are unique (up to translation) for all speeds $|\rho| \geqslant \sqrt{2 \beta}$. For $0 \leqslant|\rho|<\sqrt{2 \beta}$, there exist no monotone travelling-wave solutions of speed $\rho$.

One of the probabilistic methods for studying Eqs. (1) and (3) is via their connection to a branching Brownian motion (BBM). Consider a branching Brownian motion with drift $-\rho$, where $\rho \in \mathbb{R}$, and dyadic branching rate $\beta$; that is, a branching process where particles diffuse independently according to a Brownian motion with drift $-\rho$ and at any moment of time undergo fission with rate $\beta$ producing two particles. We shall refer to this process as a $(-\rho, \beta ; \mathbb{R})$-BBM with probabilities $\left\{P^{x}: x \in \mathbb{R}\right\}$ where $P^{x}$ is the law of the process initiated from a single particle positioned at $x$. Suppose that the configuration of space at time $t$ is given by the point process $\mathcal{X}_{t}^{-\rho}$ with points $\left\{\mathcal{Y}_{u}(t): u \in \mathcal{N}_{t}^{-\rho}\right\}$ where $\mathcal{N}_{t}^{-\rho}$ is the set of individuals alive at time $t$.

Associated with the $(-\rho, \beta ; \mathbb{R})$-BBM are the positive martingales

$$
Z_{\lambda}(t):=\sum_{u \in \mathcal{N}_{t}^{-\rho}} \mathrm{e}^{(\lambda+\rho) \mathcal{Y}_{u}(t)-\left(\frac{1}{2}\left(\lambda^{2}-\rho^{2}\right)+\beta\right) t}
$$

defined for each $\lambda \in \mathbb{R}$. It is well known that such martingales are uniformly integrable with strictly positive limits precisely when $|\lambda+\rho|<\sqrt{2 \beta}$, otherwise they have an almost surely zero limit. These martingales can be used to establish both the existence and uniqueness of the TW solutions to system (3) where, in particular, $f(x)=$ $E^{x}\left(\exp \left\{-Z_{\lambda}(\infty)\right\}\right)$ gives a representation for the TW of speed $\rho$ when $\lambda$ satisfies $\frac{1}{2}(\lambda+\rho)^{2}-\rho(\lambda+\rho)+\beta=0$. See McKean [31,32] and Neveu [33], whilst Kyprianou [27] and Harris [18] give complete expositions that are also particularly relevant for the techniques used in this paper. The latter references also include probabilistic derivations of the asymptotic behaviour of the TW solution. [Note that the martingale results for a constant drift of $\rho \in \mathbb{R}$ follow trivially from the $\rho=0$ case found in these, and subsequent, references.]

In this article we shall consider the class of solutions to the FKPP travelling-wave equation defined on $\mathbb{R}^{+}$that satisfy $f: \mathbb{R}^{+} \rightarrow[0,1], f \in C^{2}(0, \infty)$ and

$$
\begin{aligned}
& \frac{1}{2} f^{\prime \prime}-\rho f^{\prime}+\beta\left(f^{2}-f\right)=0 \quad \text { on }(0, \infty), \\
& f(0+)=1 \\
& f(\infty)=0
\end{aligned}
$$


Note that without the boundary conditions we always have that the constant functions of 0 and 1 are solutions to (4). Interestingly, solutions to the one-sided FKPP system at (4) occur at wave speeds where there are no (monotone) solutions to the FKPP travelling-wave equation on $\mathbb{R}$ :

Theorem 1. The system (4) has a unique solution if and only if $-\infty<\rho<\sqrt{2 \beta}$, in which case

$$
\lim _{x \uparrow \infty} \mathrm{e}^{-\left(\rho-\sqrt{\rho^{2}+2 \beta}\right) x} f(x)=k
$$

for some constant $k \in(0, \infty)$. Further, if $\rho \geqslant \sqrt{2 \beta}$, there is no solution to (4).

Existence, uniqueness and a weaker asymptotic result were established analytically in Pinsky [35], who himself cites Aronson and Weinberger [1]. Concerning existence, see also Watanabe [37]. Theorem 1 can be also be extracted from Kametaka [23] who also uses classical phase-plane analysis techniques (as in Coddington and Levinson [11]), although some care is required as this paper is predominantly concerned with the opposing case of $\rho \geqslant \sqrt{2 \beta}$.

In the spirit of Harris [18] and Kyprianou [27], we shall devote the first part of this article to a new proof of Theorem 1 using probabilistic means alone which, for the most part, means that we appeal either to martingale arguments, 'spine' decompositions, or fundamental properties of both branching and single-particle Brownian motion.

In contrast to the probabilistic study of travelling-waves on $\mathbb{R}$, our analysis will be concerned with a branching Brownian motion with drift $-\rho$ where particles are killed at the origin. For the purpose of the forthcoming analysis, we will construct this killed BBM, $X^{-\rho}$, from that part of the BBM $\mathcal{X}^{-\rho}$ which survives killing at the origin. Considering $X^{-\rho}$ as a subprocess of $\mathcal{X}^{-\rho}$ we shall again work with the probabilities $\left\{P^{x}: x>0\right\}$. We shall denote the configuration of particles alive at time $t$ by $\left\{Y_{u}(t): u \in N_{t}^{-\rho}\right\}$ where $N_{t}^{-\rho}$ is the number of surviving particles. In keeping with previous notation, we shall refer to this killed BBM process as a $\left(-\rho, \beta ; \mathbb{R}^{+}\right)$-BBM. We define $\zeta:=\inf \left\{t>0: N_{t}^{-\rho}=0\right\}$ to be the extinction time of the $\left(-\rho, \beta ; \mathbb{R}^{+}\right)$-BBM, then $\{\zeta=\infty\}$ is the event the process survives forever.

In Section 2, we shall briefly discuss a probabilistic technique that has recently become quite popular in the branching process literature, namely, a change of measure that induces a 'spine' decomposition of the process. In particular, if we change the measure of a $(-\rho, \beta ; \mathbb{R})$-BBM using the $Z_{\lambda}$ additive martingale, the process under the new measure can be re-constructed in law by first laying down the motion of a single particle, the so called 'spine', as a Brownian motion with modified drift $\lambda$, giving birth at an accelerated rate $2 \beta$ to independent $(-\rho, \beta ; \mathbb{R})-$ BBMs. These changes of measure and their associated spine constructions prove a key tool in our later analysis.

In Section 3 we look at some important properties of the drifting branching Brownian motion with killing at the origin. In particular, we look at the behaviour of the right-most particle, $R_{t}$, the relationship with the survival set and survival probabilities.

In Sections 4-6, we prove Theorem 1 via a sequence of smaller results. These are: non-existence of solutions to system (3) for $\rho \geqslant \sqrt{2 \beta}$ as a consequence of $P^{x}(\zeta<\infty) \equiv 1$ in the aforementioned killed BBM; existence of a solution for $-\infty<\rho<\sqrt{2 \beta}$ in the form of the (non-trivial) extinction probability $P^{x}(\zeta<\infty)$; uniqueness of travelling-waves for $-\infty<\rho<\sqrt{2 \beta}$; and finally the asymptotic result.

In Section 7, we show how our intuitive spine approach to killed BBM in Section 3 also allows us to deduce the following new result:

Lemma 2. For all $x>0$ we have

$$
\lim _{t \uparrow \infty} \frac{R_{t}}{t}=\sqrt{2 \beta}-\rho \quad \text { on }\{\zeta=\infty\}, P^{x} \text {-a.s. }
$$

With the asymptotic speed of the right-most particle in the killed BBM established and knowledge of standard $\mathrm{BBM}$, one naturally wonders if anything can be said about the asymptotic rate of growth in the numbers of particles 
travelling at slower speeds, or if any large time asymptotic can be established for the probability that the right-most particle has travelled at a faster speed. Indeed, we will answer both these questions in the remaining parts of this article, providing a more comprehensive collection of new results for killed BBM. Naturally, the close connection to standard BBM means that we can (and do) make use of some existing techniques, nevertheless adding killing at the origin yields some significant new problems.

The central role of the $Z_{\lambda}$ additive martingales in the study of branching Brownian motion is long established and, as already indicated, we can also exploit them for killed BBM. However, we also introduce the (positive) additive martingales $W_{\lambda}$ :

Lemma 3. For each $\lambda>0$, the process

$$
W_{\lambda}(t):=\sum_{u \in N_{t}^{-\rho}}\left(1-\mathrm{e}^{-2 \lambda Y_{u}(t)}\right) \mathrm{e}^{(\lambda+\rho) Y_{u}(t)-\left(\frac{1}{2}\left(\lambda^{2}-\rho^{2}\right)+\beta\right) t},
$$

defines a martingale for the $\left(-\rho, \beta ; \mathbb{R}^{+}\right)-B B M$.

These martingales not only prove to be a useful tool, they appear fundamental to the study of the killed BBM. In Section 8, we discuss their convergence properties and how they can be used to deduce growth rates of particles moving at speeds $\lambda<\sqrt{2 \beta}-\rho$.

Theorem 4. $W_{\lambda}$ is a uniformly integrable martingale if both $\rho<\sqrt{2 \beta}$ and $\lambda \in(0, \sqrt{2 \beta}-\rho)$, otherwise $W_{\lambda}$ has a $P^{x}$-almost-sure zero limit.

Whenever $W_{\lambda}$ is uniformly integrable, $\left\{W_{\lambda}(\infty)>0\right\}$ and $\{\zeta=\infty\}$ agree up to a $P^{x}$-null set.

Define the counting function

$$
N_{t}^{-\rho}(a, b):=\sum_{u \in N_{t}^{-\rho}} \mathbf{1}_{\left\{Y_{u}(t) \in(a, b)\right\}}
$$

for the number of particles found in the interval $(a, b)$ at time $t$.

Theorem 5. For $x>0$, under each $P^{x}$ law, the limit

$$
G(\lambda):=\lim _{t \rightarrow \infty} t^{-1} \ln N_{t}^{-\rho}(\lambda t, \infty)
$$

exists almost surely and is given by

$$
G(\lambda)= \begin{cases}\Delta(\lambda), & \text { if } 0<\lambda<\sqrt{2 \beta}-\rho \text { and }\{\zeta=\infty\} \\ -\infty, & \text { otherwise }\end{cases}
$$

where $\Delta(\lambda):=\beta-\frac{1}{2}(\lambda+\rho)^{2}$.

Note: we gain the right-most particle speed of Lemma 2 as a corollary.

These results are both new, although we make use of the techniques from Git et al. [16] in proving Theorem 5. Also see Kesten [24] where some related questions on survival probabilities and population growth-rates in fixed subsets of $\mathbb{R}$ for a similar branching Brownian motion with an absorbing barrier are considered.

In Section 9, we conclude by investigating the probability that the right-most particle has travelled at faster speeds than usual, proving the following new result:

Theorem 6. For $\lambda>\sqrt{2 \beta}-\rho$ and all $x>0, \theta \geqslant 0$, there exists a constant $C>0$ such that

$$
\lim _{t \rightarrow \infty} P^{x}\left(R_{t} \geqslant \lambda t+\theta\right) \frac{\sqrt{2 \pi t}}{1-\mathrm{e}^{-2 \lambda x}} \mathrm{e}^{-(\lambda+\rho)(x-\theta)+\left(\frac{1}{2}(\lambda+\rho)^{2}-\beta\right) t}=C,
$$


or equivalently,

$$
P^{x}\left(R_{t}>\lambda t+\theta\right) \underset{t \rightarrow \infty}{\sim}(\lambda+\rho) C \times E^{x}\left(N_{t}^{-\rho}(\lambda t+\theta, \infty)\right) .
$$

Considering particles with spatial position $Y_{u}(t)>\lambda t+\theta$, for $\lambda>\sqrt{2 \beta}-\rho$, a Yaglom-type result also holds:

Theorem 7. For $\lambda>\sqrt{2 \beta}-\rho$ there is a probability distribution $\left(\Pi_{i}\right)_{i} \geqslant 1$ defined on $\mathbb{N}$ such that

$$
\lim _{t \rightarrow \infty} P^{x}\left(N_{t}^{-\rho}(\lambda t, \infty)=i \mid N_{t}^{-\rho}(\lambda t,+\infty)>0\right)=\Pi_{i},
$$

and this distribution has (finite) expectation equal to $1 /(\lambda+\rho) C$.

Finally, we can see the fundamental nature of the $W_{\lambda}$ martingale that we introduced into the killed BBM story: it appears as the Radon-Nikodým derivative linking $P^{x}$ with the limit-law of the conditioned process.

Theorem 8. For $\lambda>\sqrt{2 \beta}-\rho, s \in(0, \infty)$ fixed, and $A \in \mathcal{F}_{s}$,

$$
P^{x}\left(A \mid N_{t+s}^{-\rho}(\lambda(t+s), \infty)>0\right) \underset{t \rightarrow+\infty}{\longrightarrow} E^{x}\left(\mathbf{1}_{A} W_{\lambda}(s)\right) .
$$

Chauvin and Rouault [9] proved analogous results to Theorems 6-8 in the context of standard BBM. Although guided by their approach when we prove Theorem 6 , there are a number of complications caused by the killing at the origin. However, once these additional difficulties are overcome and we have proven Theorem 6, the BBM proofs of Chauvin and Rouault [9] adapt almost unchanged for Theorems 7 and 8 and we omit any details here.

\section{Spine decompositions for BBM}

In this section, we briefly recall some changes of measure and their associated spine decompositions that will be key tools in later analysis.

When $|\lambda+\rho|<\sqrt{2 \beta}$ one can define an equivalent change of measure on the probability space of the $(-\rho, \beta ; \mathbb{R})$ BBM via

$$
\left.\frac{\mathrm{d} \pi_{\lambda}^{x}}{\mathrm{~d} P^{x}}\right|_{\mathcal{F}_{t}}=\frac{Z_{\lambda}(t)}{Z_{\lambda}(0)}=\mathrm{e}^{-(\lambda+\rho) x} Z_{\lambda}(t) .
$$

Under $\pi_{\lambda}^{x}$ the path of the $(-\rho, \beta ; \mathbb{R})$-BBM can be reconstructed pathwise in the following way:

- starting from position $x$, the initial ancestor diffuses according to a Brownian motion with drift $\lambda$;

- at rate $2 \beta$ the particle undergoes fission producing two particles;

- one of these particles is selected at random with probability one half;

- this chosen particle repeats stochastically the behaviour of their parent;

- the other particle initiates from its birth position an independent copy of a $(-\rho, \beta ; \mathbb{R})$-BBM with law $P$, and so on.

The selected line of descent is referred to as the spine. Thus, the spine moves as a Brownian motion with drift $\lambda$, giving birth at an accelerated rate $2 \beta$ along its path to independent $(-\rho, \beta ; \mathbb{R})$-BBMs.

The martingale $W_{\lambda}$ can be used to change measure in a similar fashion to yield a spine that is conditioned to avoid the origin. Before giving the details we shall prove Lemma 3. 
Proof of Lemma 3. Let $Y(t)$ be a Brownian motion started at $x>0$ with drift $\lambda$ under the measure $\mathbb{P}_{\lambda}^{x}$. Defining $\tau_{0}:=\inf \{t \geqslant 0: Y(t)=0\}$, we have from the 'many-to-one' lemma that for measurable $f$

$$
E^{x}\left(\sum_{u \in N_{t}} f\left(Y_{u}(t)\right)\right)=\mathrm{e}^{\beta t} \mathbb{E}_{-\rho}^{x}\left(f\left(Y_{t}\right) ; \tau_{0}>t\right) .
$$

(See, for example, Hardy and Harris [17] or equivalently consider the single particle representation of the linear semigroup associated with the $\left(-\rho, \beta, \mathbb{R}^{+}\right)$-BBM.) Using the latter equality together with Girsanov change of measure it follows that

$$
\begin{aligned}
E^{x}\left(W_{\lambda}(t)\right) & =\mathrm{e}^{-\frac{1}{2}\left(\lambda^{2}-\rho^{2}\right) t} \mathbb{E}_{-\rho}^{x}\left(\left(1-\mathrm{e}^{-2 \lambda Y_{t}}\right) \mathrm{e}^{(\lambda+\rho) Y_{t}} ; \tau_{0}>t\right) \\
& =\mathbb{P}_{\lambda}^{x}\left(\left(1-\mathrm{e}^{-2 \lambda Y_{t}}\right) ; \tau_{0}>t\right) \mathrm{e}^{(\lambda+\rho) x} \\
& =\left(1-\mathrm{e}^{-2 \lambda x}\right) \mathrm{e}^{(\lambda+\rho) x} .
\end{aligned}
$$

Note the last equality is a simple consequence of useful the fact that $\mathbb{P}_{\lambda}^{x}\left(\tau_{0}=\infty\right)=1-\mathrm{e}^{-2 \lambda x}$ is a scale function for $Y$ killed at the origin under $\mathbb{P}_{\lambda}^{x}$.

To complete the proof, apply the branching property to note that

$$
E^{x}\left(W_{\lambda}(t+s) \mid \mathcal{F}_{t}\right)=\sum_{u \in N_{t}^{-\rho}} E^{x}\left(W_{\lambda}^{(u)}(s) \mid \mathcal{F}_{t}\right) \mathrm{e}^{-\left(\frac{1}{2}\left(\lambda^{2}-\rho^{2}\right)+\beta\right) t}
$$

where given $\mathcal{F}_{t}$, each of the terms $W_{\lambda}^{(u)}(s)$ are independent copies of $W_{\lambda}(s)$ under $P^{Y_{u}(t)}$. The conclusion of the previous paragraph now completes the proof.

We define a measure $\mathbb{Q}_{\lambda}$ by

$$
\left.\frac{\mathrm{d} \mathbb{Q}_{\lambda}^{x}}{\mathrm{~d} P^{x}}\right|_{\mathcal{F}_{t}}=\frac{W_{\lambda}(t)}{W_{\lambda}(0)}
$$

and it can be shown that under $\mathbb{Q}_{\lambda}$ the BBM can be constructed path-wise as under $\pi_{\lambda}^{x}$, except that the spine now moves like a Brownian motion with drift $\lambda$ that is additionally conditioned to avoid the origin.

For a detailed setup and notation for these changes of measure and spine decompositions in branching Brownian motion the reader should refer to the recent new approach presented in Hardy and Harris [17], in addition to Kyprianou [27] and Chauvin and Rouault [9]. More generally, similar changes of measure for other types of branching processes have become increasingly common in the study of classical and modern branching processes; in particular, the reader is referred to Lyons et al. [29] and Lyons [30]. Champneys et al. [7], Harris and Williams [19], Olofsson [34], Athreya [2], Kyprianou and Rahimzadeh Sani [28], Biggins and Kyprianou [3], Engländer and Kyprianou [13] and Kuhlbusch [26] also provide further examples of their use.

\section{Killed branching Brownian motion}

It will turn out that the existence and uniqueness result in Theorem 1 can be proved probabilistically by analysing the $\left(-\rho, \beta ; \mathbb{R}^{+}\right)$-BBM and in particular the behaviour of the position of its right most particle, defined by

$$
R_{t}=\sup \left\{Y_{u}(t): u \in N_{t}^{-\rho}\right\}
$$

on $\{\zeta=\infty\}$ and zero otherwise.

Theorem 9. We have for all $x>0$ and $\rho \in \mathbb{R}$,

$$
\limsup _{t \uparrow \infty} R_{t}=\infty \quad \text { on }\{\zeta=\infty\}, P^{x} \text {-a.s. }
$$


Proof. Let us suppose that $Y=\{Y(t): t \geqslant 0\}$ is a Brownian motion with drift $-\rho$ and probabilities $\left\{\mathbb{P}_{-\rho}^{x}: x \in \mathbb{R}\right\}$ and let $\tau_{0}=\inf \{t \geqslant 0: Y(t)=0\}$. Note that

$$
P^{x}\left(\zeta<\infty \mid \mathcal{F}_{t}\right) \geqslant \prod_{u \in N_{t}^{-\rho}} \mathbb{P}_{-\rho}^{Y_{u}(t)}\left(\tau_{0}<\mathbf{e}_{\beta}\right)=\prod_{u \in N_{t}^{-\rho}} \mathbb{E}_{-\rho}^{Y_{u}(t)}\left(\mathrm{e}^{-\beta \tau_{0}}\right)
$$

where $\mathbf{e}_{\beta}$ is an exponential variable independent of $Y$ having rate $\beta$. The last inequality follows on account of the fact that extinction would follow if each of the individuals alive at time $t$ would hit the origin before splitting. Setting $\alpha=\rho-\sqrt{\rho^{2}+2 \beta}<0$, standard expressions for the one sided exit problem for Brownian motion (see, for example, Borodin and Salminen [4]), imply that for all $x>0$

$$
P^{x}\left(\zeta<\infty \mid \mathcal{F}_{t}\right) \geqslant \prod_{u \in N_{t}^{-\rho}} \mathrm{e}^{\alpha Y_{u}(t)}=\exp \left(\alpha \sum_{u \in N_{t}^{-\rho}} Y_{u}(t)\right)
$$

On $\{\zeta=\infty\}$ it is clear that the left hand side converges to zero and hence for all $x>0$

$$
\lim _{t \uparrow \infty} \sum_{u \in N_{t}^{-\rho}} Y_{u}(t)=\infty \quad \text { on }\{\zeta=\infty\} P^{x} \text {-a.s. }
$$

Now let $\Gamma_{z}$ be the event that the $\left(-\rho, \beta ; \mathbb{R}^{+}\right)$-BBM is contained entirely in the strip $(0, z)$. For the process $Y$ define the stopping time $\tau_{z}=\inf \{t \geqslant 0: Y(t)=z\}$. We have for $0<x<z$

$$
P^{x}\left(\Gamma_{z} \mid \mathcal{F}_{t}\right) \leqslant \prod_{u \in N_{t}^{-\rho}} \mathbb{P}_{-\rho}^{Y_{u}(t)}\left(\tau_{0}<\tau_{z}\right)
$$

on $Y_{u}(t) \in(0, z)$ for $u \in N_{t}^{-\rho}$. This inequality follows from the fact that $\Gamma_{z}$ implies that the spatial path of each of the lines of descent emanating from the configuration at time $t$ must hit the origin before hitting $z$. First consider the case that $-\infty<\rho<0$. In this case we can write from (11) on the event that $Y_{u}(t) \in(0, z)$ for each $u \in N_{t}^{-\rho}$

$$
P^{x}\left(\Gamma_{z} \mid \mathcal{F}_{t}\right) \leqslant \prod_{u \in N_{t}^{-\rho}} \mathrm{e}^{-|\rho| Y_{u}(t)} \frac{\sinh |\rho|\left(z-Y_{u}(t)\right)}{\sinh |\rho| z} \leqslant \exp \left(-|\rho| \sum_{u \in N_{t}^{-\rho}} Y_{u}(t)\right) \rightarrow 0
$$

on the event $\{\zeta=\infty\}$ as $t$ tends to infinity. Now consider the case that $\rho>0$. It follows by again using classical results for the two sided exit problem that on the event that $Y_{u}(t) \in(0, z)$ for each $u \in N_{t}^{-\rho}$

$$
\begin{aligned}
P^{x}\left(\Gamma_{z} \mid \mathcal{F}_{t}\right) & \leqslant \prod_{u \in N_{t}^{-\rho}}\left[1-\left(\frac{\mathrm{e}^{-\rho z}}{\sinh \rho z}\right) \mathrm{e}^{\rho Y_{u}(t)} \sinh \rho Y_{u}(t)\right] \\
& \leqslant \exp \left(-\left(\frac{\mathrm{e}^{-\rho z}}{\sinh \rho z}\right) \sum_{u \in N_{t}^{-\rho}} Y_{u}(t)\right) \rightarrow 0
\end{aligned}
$$

where we have used the inequalities $\mathrm{e}^{-x} \sinh x \leqslant x$ and $1-x \leqslant \mathrm{e}^{-x}$. The latter exponential tends to zero on the event $\{\zeta=\infty\}$ as $t$ tends to infinity. Finally for the case that $\rho=0$, on $Y_{u}(t) \in(0, z)$ for $u \in N_{t}^{-\rho}$

$$
P^{x}\left(\Gamma_{z} \mid \mathcal{F}_{t}\right) \leqslant \prod_{u \in N_{t}^{-\rho}}\left(1-\frac{Y_{u}(t)}{z}\right) \leqslant \exp \left(-\frac{1}{z} \sum_{u \in N_{t}^{-\rho}} Y_{u}(t)\right) \rightarrow 0
$$

on the event $\{\zeta=\infty\}$ as $t$ tends to infinity. In conclusion, for any $z>0, P^{x}\left(R_{t}>z\right.$ i.o. $\left.\mid \zeta=\infty\right)=1$ and the statement of the theorem holds.

Theorem 10. If $\rho \geqslant \sqrt{2 \beta}$ then $P^{x}(\zeta<\infty)=1$ for all $x>0$. 
Proof. Suppose that $\mathcal{R}_{t}$ is the position of the right most particle in a $(-\rho, \beta ; \mathbb{R})$-BBM. It is well known (see Neveu [33], Harris [18] or Kyprianou [27]) that the 'critical' martingale $Z_{\sqrt{2 \beta}-\rho}(t) \rightarrow 0$ a.s., from which it is easy to deduce that

$$
\lim _{t \uparrow \infty}\left\{\mathcal{R}_{t}-(\sqrt{2 \beta}-\rho) t\right\}=-\infty \quad \text { a.s. }
$$

From our construction of the $\left(-\rho, \beta ; \mathbb{R}^{+}\right)$-BBM, extinction of this process is guaranteed when the right most particle in the $(-\rho, \beta ; \mathbb{R})$-BBM drifts to $-\infty$. Thus, when $\rho \geqslant \sqrt{2 \beta}$, this happens with probability one.

Theorem 11. If $-\infty<\rho<\sqrt{2 \beta}$ then for each $x>0$ and $\lambda \in(0, \sqrt{2 \beta}-\rho)$

(i) $E^{x}\left(Z_{\lambda}(\infty) ; \liminf _{t \geqslant 0} R_{t} / t \geqslant \lambda\right)=\pi_{\lambda}^{x}\left(\liminf _{t} \geqslant 0 R_{t} / t \geqslant \lambda\right) \geqslant 1-\mathrm{e}^{-2 \lambda x}$,

(ii) $P^{x}(\zeta<\infty) \in(0,1)$,

(iii) $\lim _{x \downarrow 0} P^{x}(\zeta<\infty)=1$, and

(iv) $\lim _{x \uparrow \infty} P^{x}(\zeta=\infty)=\lim _{x \uparrow \infty} P^{x}\left(\liminf _{t \uparrow \infty} R_{t} / t \geqslant \lambda ; \zeta=\infty\right)=1$.

Proof. (i) Recall that when $|\lambda+\rho|<\sqrt{2 \beta}$ (and in particular when $\lambda \in(0, \sqrt{2 \beta}-\rho)$ ), under the measure $\pi_{\lambda}^{x}$ (defined in Section 2) a branching Brownian motion with drift $-\rho$ and branching rate $\beta$ has one line of descent, the spine, which has an exceptional drift $\lambda$. The probability that this spine never meets the origin is the probability that a Brownian motion started from $x>0$ and with drift $\lambda$ has an all time infimum which is strictly positive; and this is well known to be $1-\exp \{-2 \lambda x\}$. Suppose we write $\xi=\left\{\xi_{t}: t \geqslant 0\right\}$ for the spatial path of any surviving line of descent in $X^{-\rho}$ then we have established that

$$
E^{x}\left(Z_{\lambda}(\infty) ; \zeta=\infty \text { and } \exists \xi \text { in } X^{-\rho} \text { such that } \lim _{t \uparrow \infty} \frac{\xi_{t}}{t}=\lambda\right) \geqslant 1-\exp \{-2 \lambda x\} .
$$

Now note that

$$
\left\{\zeta=\infty \text { and } \exists \xi \text { in } X^{-\rho} \text { such that } \lim _{t \uparrow \infty} \frac{\xi_{t}}{t}=\lambda\right\} \subseteq\left\{\liminf _{t \uparrow \infty} R_{t} / t \geqslant \lambda\right\}
$$

and hence the statement of part (i) now follows.

(ii) To prove that $P^{x}(\zeta<\infty)>0$, note that there is a strictly positive probability that the initial ancestor in the process $X^{-\rho}$ hits the origin before reproducing thus resulting in extinction. To prove that $P^{x}(\zeta<\infty)<1$ or equivalently $P^{x}(\zeta=\infty)>0$, recall from part (i) that under $\pi_{\lambda}^{x}$ the probability that the ( $\lambda$-drifting) spine in a branching Brownian motion with does not meet the origin is strictly positive. This is implies that $E^{x}\left(Z_{\lambda}(\infty) ; \zeta=\right.$ $\infty)>0$ and since $P^{x}\left(Z_{\lambda}(\infty)>0\right)=1$ it follows that $P^{x}(\zeta=\infty)>0$.

(iii) Since extinction in a finite time is guaranteed if the original ancestor is killed before reproducing,

$$
P^{x}(\zeta<\infty) \geqslant \mathbb{P}_{-\rho}^{x}\left(\tau_{0}<\mathbf{e}_{\beta}\right)=\mathrm{e}^{-\left(\sqrt{\rho^{2}+2 \beta}-\rho\right) x} \rightarrow 1
$$

as $x \rightarrow 0$. (Recall that $\tau_{0}=\inf \{t \geqslant 0: Y(t)=0\}$ and $\mathbf{e}_{\beta}$ is exponentially distributed with parameter $\beta$ and independent of the Brownian motion $\left.\left(Y, \mathbb{P}_{-\rho}^{x}\right)\right)$.

(iv) Note that $P^{x}\left(\liminf _{t \uparrow \infty} R_{t} / t \geqslant \lambda ; \zeta=\infty\right)$ is an increasing sequence in $x$ and therefore has a limit. Suppose this limit is not equal to one then since it was shown in part (i) of the proof that

$$
\lim _{x \uparrow \infty} E^{x}\left(Z_{\lambda}(\infty) ; \liminf _{t \geqslant 0} R_{t} / t \geqslant \lambda\right)=1
$$

there is a contradiction since for all $x>0$

$$
P^{x}\left(Z_{\lambda}(\infty)>0\right)=1 \quad \text { and } \quad E^{x}\left(Z_{\lambda}(\infty)\right)=1
$$

Finally noting that $P^{x}(\zeta=\infty) \geqslant P^{x}\left(\liminf _{t \uparrow \infty} R_{t} / t \geqslant \lambda, \zeta=\infty\right)$ the proof is complete. 


\section{Non-existence for $\rho \geqslant \sqrt{2 \beta}$}

Theorem 12. No travelling-wave solutions to (4) exist for $\rho \geqslant \sqrt{2 \beta}$.

Proof. Now suppose that $f$ is a solution to (4). It follows that for all $x>0 \prod_{u \in N_{t}^{-\rho}} f\left(Y_{u}(t)\right)$ is a martingale which converges almost surely and in $L^{1}\left(P^{x}\right)$. We have seen in Theorem 10 that if $\rho \geqslant \sqrt{2 \beta}, P^{x}(\zeta<\infty)=1$ for all $x>0$ and hence

$$
\lim _{t \uparrow \infty} \prod_{u \in N_{t}^{-\rho}} f\left(Y_{u}(t)\right)=1
$$

almost surely, implying that $f=1$; that is to say, there is no non-trivial solution.

\section{Existence and uniqueness for $-\infty<\rho<\sqrt{2 \beta}$}

Theorem 13. Travelling-waves to (4) exist and are unique for $-\infty<\rho<\sqrt{2 \beta}$. Further, the unique solution can be represented by the extinction probability for the $\left(-\rho, \beta ; \mathbb{R}^{+}\right)-B B M$, that is

$$
f(x)=P^{x}(\zeta<\infty) .
$$

Remark 14. The representation (12) trivially shows that the unique solution to (4) is strictly monotone decreasing, although this wasn't an initial restriction. Indeed, one could assume monotonicity instead of $f(\infty)=0$ and again reach the same conclusions. Also note, that one might naively try to extended this solution to produce a travellingwave of speed $\rho<\sqrt{2 \beta}$ on the whole of $\mathbb{R}$, but such a solution would clearly fail to satisfy Eq. (3) at a single point (due to a discontinuity in the first derivative at the origin).

Proof. Define $p(x):=P^{x}(\zeta<\infty)$ for $x \geqslant 0$. From Theorem 11, we have $p(x) \in(0,1)$ for each $x>0$, $\lim _{x \uparrow \infty} p(x)=0, \lim _{x \downarrow 0} p(x)=1$ and, in addition, $p(0)=1$ because of instantaneous killing.

An application of the Branching Markov Property (cf. Chauvin [8]) together with the tower property of conditional expectation gives

$$
p(x)=E^{x}\left(P^{x}\left(\zeta<\infty \mid \mathcal{F}_{t}\right)\right)=E^{x}\left(\prod_{u \in N_{t}^{-\rho}} p\left(Y_{u}(t)\right)\right) .
$$

As this equality holds for all $x, t>0$, one can easily see that $\prod_{u \in N_{t}^{-\rho}} p\left(Y_{u}(t)\right)$ is a martingale which converges almost surely and in $L^{1}\left(P^{x}\right)$. Note that on $\{\zeta<\infty\}$ it is clear that the martingale limit is equal to 1 - the empty product. Note however that this martingale cannot be identically equal to 1 because its mean, $p(x)$, is strictly less than one.

An application of Kolmogorov's backwards equations (cf. Champneys et al. [7] or Dynkin [12, Theorem II.3.1]) thus yields that $p$ belongs to $C^{2}(0, \infty)$ and is a solution to the ODE in (4).

For uniqueness, suppose that $f$ is a solution to (4) when $-\infty<\rho<\sqrt{2 \beta}$. Again we can construct a positive martingale $M_{t}:=\prod_{u \in N_{t}^{-\rho}} f\left(Y_{u}(t)\right)$ which is bounded, hence uniformly integrable. Clearly $M_{\infty}=1$ on $\{\zeta<\infty\}$. Further, Theorem 9 gives $\lim \sup _{t \uparrow \infty} R_{t}=\infty$ on $\{\zeta=\infty\}$ a.s. and, since $f(+\infty)=0$,

$$
\begin{aligned}
M_{\infty}=\lim _{t \uparrow \infty} \prod_{u \in N_{t}^{-\rho}} f\left(Y_{u}(t)\right) & =\liminf _{t \uparrow \infty} \prod_{u \in N_{t}^{-\rho}} f\left(Y_{u}(t)\right) \\
& \leqslant \liminf _{t \uparrow \infty} f\left(R_{t}\right) \\
& \leqslant f\left(\limsup _{t \uparrow \infty} R_{t}\right),
\end{aligned}
$$


we can identify the limit as $M_{\infty}=\mathbf{1}_{\{\zeta<\infty\}}$ a.s. Finally,

$$
f(x)=E^{x}\left(M_{0}\right)=E^{x}\left(M_{\infty}\right)=P^{x}(\zeta<\infty)=p(x)
$$

and uniqueness follows.

\section{Asymptotic when $-\infty<\rho<\sqrt{2 \beta}$}

In this section we determine the asymptotic for the solution to the one-sided FKPP system (4). As a first step, the following lemma shows that the unique solution decays exponentially for sufficiently large $y$ and will prove very useful:

Lemma 15. Let $f$ be the unique solution of the system (4) when $-\infty<\rho<\sqrt{2 \beta}$. Let $x_{0}>0$ and define $\mu:=$ $\sqrt{\rho^{2}+2 \beta\left(1-f\left(x_{0}\right)\right)}-\rho>0$. Then

$$
f(y) \leqslant\left(f\left(x_{0}\right) \mathrm{e}^{\mu x_{0}}\right) \mathrm{e}^{-\mu y}
$$

for all $y>x_{0}$.

Proof. Recall that $Y$ is a Brownian motion with drift $-\rho$ starting from $x>0$ under $\mathbb{P}_{-\rho}^{x}$ and recall that for any $z \geqslant 0, \tau_{z}:=\inf \left\{t: Y_{t}=z\right\}$. Itô's formula implies that,

$$
M_{t}:=f\left(Y_{t \wedge \tau_{0}}\right) \exp \left(\beta \int_{0}^{t \wedge \tau_{0}}\left(f\left(Y_{s}\right)-1\right) \mathrm{d} s\right)
$$

is a $\mathbb{P}_{-\rho}^{x}$-local martingale and, since $0 \leqslant f \leqslant 1$, it is actually a bounded martingale. Suppose that $y>x_{0}$. Since $\tau_{x_{0}}<\infty$ a.s. under $\mathbb{P}_{-\rho}^{y}$, the optional stopping theorem and the monotonicity of $f$ (see remark after Theorem 13) yields

$$
f(y)=\mathbb{E}_{-\rho}^{y}\left\{f(x) \exp \left(\beta \int_{0}^{\tau_{x_{0}}}\left(f\left(Y_{s}\right)-1\right) \mathrm{d} s\right)\right\} \leqslant f(x) \mathbb{E}_{-\rho}^{y}\left(\mathrm{e}^{\beta\left(f\left(x_{0}\right)-1\right) \tau_{x_{0}}}\right) .
$$

A well-known result (see, for example, Borodin and Salminen [4]) gives

$$
\mathbb{E}_{-\rho}^{y}\left(\mathrm{e}^{\beta\left(f\left(x_{0}\right)-1\right) \tau_{x_{0}}}\right)=\mathrm{e}^{-\mu\left(y-x_{0}\right)},
$$

where $\mu:=\sqrt{\rho^{2}+2 \beta\left(1-f\left(x_{0}\right)\right)}-\rho>0$. Thus, inequality (14) becomes

$$
f(y) \leqslant f\left(x_{0}\right) \mathrm{e}^{-\mu\left(y-x_{0}\right)}
$$

as required.

As a corollary, we gain an alternative and straightforward probabilistic proof of the weaker asymptotic result found in Pinsky [35] that first motivated this article:

Corollary 16. When it exists, the solution to the system (4) satisfies

$$
\lim _{x \uparrow \infty} \frac{1}{x} \log f(x)=\rho-\sqrt{\rho^{2}+2 \beta}<0 .
$$




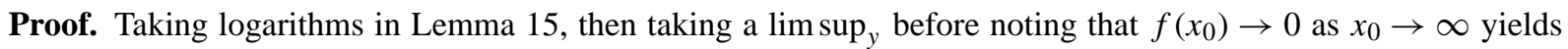
the required upper bound.

To prove the lower bound, recall that (14) is a martingale and hence for $y>0$, remembering that $f(x) \in[0,1]$ with $f(0)=1$, we have

$$
f(y)=\mathbb{E}_{-\rho}^{y}\left\{\exp \left(\beta \int_{0}^{\tau_{0}}\left(f\left(Y_{s}\right)-1\right) \mathrm{d} s\right)\right\} \geqslant \mathbb{E}_{-\rho}^{y}\left(\mathrm{e}^{-\beta \tau_{0}}\right)=\mathrm{e}^{-\left(\sqrt{\rho^{2}+2 \beta}-\rho\right) y},
$$

yielding the required lower bound.

As another corollary to Lemma 15 , we can find an exponentially decaying bound for $f$ valid on the whole of $(0, \infty)$. This is of importance in the proof of the strong asymptotic of Theorem 1 .

Corollary 17. Let $f$ be the unique solution of the system (4) when $-\infty<\rho<\sqrt{2 \beta}$. Given any $K>1$, there exists $a \kappa>0$ such that

$$
f(y) \leqslant K \mathrm{e}^{-\kappa y}
$$

for all $y \geqslant 0$.

Proof. For $K>1$, choose $x_{0}>0$ such that $K=\mathrm{e}^{\mu x_{0}}$. Note that $f\left(x_{0}\right) \in(0,1)$ and then set

$$
\kappa=\sqrt{\rho^{2}+2 \beta\left(1-f\left(x_{0}\right)\right)}-\rho>0 .
$$

Lemma 15 says that $f(y) \leqslant K \mathrm{e}^{-\kappa y}$ for all $y \geqslant x_{0}$. Also, since $0 \leqslant f \leqslant 1$ and for $y<x_{0}$ we have $K \mathrm{e}^{-\kappa y}>1$, we trivially have $f(y) \leqslant K \mathrm{e}^{-\kappa y}$ for all $y \leqslant x_{0}$.

We now extend the analysis to prove the stronger asymptotic of Theorem 1. Crucial to the argument will be the following proposition which we shall prove at the end of this section.

Proposition 18. With $\tilde{\rho}:=\sqrt{\rho^{2}+2 \beta}, x>0$ and $f(x)$ the unique travelling-wave at speed $-\infty<\rho<\sqrt{2 \beta}$,

$$
\lim _{x \rightarrow \infty} \mathbb{E}_{-\tilde{\rho}}^{x} \exp \left(\beta \int_{0}^{\tau_{0}} f\left(Y_{s}\right) \mathrm{d} s\right)<+\infty
$$

Proof of Theorem 1 (asymptotics). Working with the change of measure

$$
\left.\frac{\mathrm{d} \mathbb{P}_{\lambda-\rho}^{x}}{\mathrm{~d} \mathbb{P}_{-\rho}^{x}}\right|_{\mathcal{F}_{t}}=\mathrm{e}^{\lambda\left(Y_{t}+\rho t-x\right)-\frac{1}{2} \lambda^{2} t}
$$

for $\lambda \in \mathbb{R}$ and $x>0$, we have from the martingale property at (14) that

$$
\mathrm{e}^{-\lambda x} f(x)=\mathbb{E}_{-\rho}^{x}\left\{\mathrm{e}^{-\lambda Y_{t \wedge \tau_{0}}} f\left(Y_{t \wedge \tau_{0}}\right) \mathrm{e}^{\beta \int_{0}^{t \wedge \tau_{0}} f\left(Y_{s}\right) \mathrm{d} s} \mathrm{e}^{\lambda\left(Y_{t \wedge \tau_{0}}-x\right)-\beta\left(t \wedge \tau_{0}\right)}\right\}
$$

Choosing $\lambda=\alpha:=\rho-\sqrt{\rho^{2}+2 \beta}<0$, so that $\beta+\rho \lambda=\frac{1}{2} \lambda^{2}$, and defining $v(x):=\mathrm{e}^{-\alpha x} f(x)$ and $\tilde{\rho}:=$ $\sqrt{\rho^{2}+2 \beta}>0$, this yields

$$
v(x)=\mathbb{E}_{-\tilde{\rho}}^{x}\left\{v\left(Y_{t \wedge \tau_{0}}\right) \exp \left(\beta \int_{0}^{t \wedge \tau_{0}} f\left(Y_{S}\right) \mathrm{d} s\right)\right\},
$$


whence

$$
v\left(Y_{t \wedge \tau_{0}}\right) \exp \left(\beta \int_{0}^{t \wedge \tau_{0}} f\left(Y_{s}\right) \mathrm{d} s\right)
$$

is a $\mathbb{P}_{-\tilde{\rho}}$ martingale which is positive and therefore convergent. As $\tau_{0}<\infty$ a.s. $\mathbb{P}_{-\tilde{\rho}}$ we also have $v\left(Y_{t \wedge \tau_{0}}\right) \rightarrow 1$ a.s. under $\mathbb{P}_{-\tilde{\rho}}^{x}$, but $v$ is not (yet) known to be a bounded function so we cannot immediately conclude that this martingale is uniformly integrable. However, using the change of measure

$$
\left.\frac{\mathrm{d} \mathbb{P}_{-\tilde{\rho}}^{x}}{\mathrm{~d} \mathbb{P}_{-\rho}^{x}}\right|_{\mathcal{F}_{\tau_{0}}}=\mathrm{e}^{\left(\sqrt{\rho^{2}+2 \beta}-\rho\right) x-\beta \tau_{0}}
$$

(which is possible because $\exp \left\{-\left(\sqrt{\rho^{2}+2 \beta}-\rho\right)\left(Y_{t \wedge \tau_{0}}-x\right)-\beta\left(t \wedge \tau_{0}\right)\right\}$ is a uniformly integrable martingale) we may transform (15) to

$$
v(x)=\mathbb{E}_{-\tilde{\rho}}^{x}\left\{\exp \left(\beta \int_{0}^{\tau_{0}} f\left(Y_{s}\right) \mathrm{d} s\right)\right\}
$$

and hence the $\mathbb{P}_{-\tilde{\rho}}^{x}$ martingale in (17) is uniformly integrable. Note that from (18) it is clear that $v$ is monotone increasing in $x$ and hence its limit exists as $x$ tends to infinity.

All that remains is to prove that $v$ converges to a finite limit as $x$ tends to infinity, which is precisely Proposition 18. Thus

$$
v(x):=f(x) \mathrm{e}^{-\alpha x} \uparrow k \in(0, \infty) \quad \text { as } x \rightarrow \infty .
$$

Hence $f(x)$ asymptotically looks like the decaying solution of

$$
\frac{1}{2} f^{\prime \prime}-\rho f^{\prime}-\beta f=0
$$

that is, the linearization of Eq. (3) about the origin.

Proof of Proposition 18. Recall that $\tilde{\rho}=\sqrt{\rho^{2}+2 \beta}$ and for $y>0$

$$
\mathbb{E}_{-\tilde{\rho}}^{y}\left(\mathrm{e}^{\gamma \tau_{0}}\right)=\mathrm{e}^{\left(\tilde{\rho}-\sqrt{\tilde{\rho}^{2}-2 \gamma}\right) y}
$$

provided that $2 \gamma<\tilde{\rho}^{2}$ (in particular, this holds for all $\gamma \leqslant \beta$ ).

Note that for any $y>0$, since $f \in[0,1]$, we have

$$
\mathbb{E}_{-\tilde{\rho}}^{y} \exp \left(\beta \int_{0}^{\tau_{0}} f\left(Y_{s}\right) \mathrm{d} s\right) \leqslant \mathbb{E}_{-\tilde{\rho}}^{y}\left(\mathrm{e}^{\beta \tau_{0}}\right)=\mathrm{e}^{\left(\sqrt{\rho^{2}-2 \beta}-\rho\right) y}<\infty,
$$

and for any $y_{0}>y_{1}>0$, the strong Markov property gives

$$
\mathbb{E}_{-\tilde{\rho}}^{y_{0}}\left(\mathrm{e}^{\beta \int_{0}^{\tau_{0}} f\left(Y_{s}\right) \mathrm{d} s}\right)=\mathbb{E}_{-\tilde{\rho}}^{y_{0}}\left(\mathrm{e}^{\beta \int_{0}^{\tau_{y_{1}}} f\left(Y_{s}\right) \mathrm{d} s}\right) \mathbb{E}_{-\tilde{\rho}}^{y_{1}}\left(\mathrm{e}^{\beta \int_{0}^{\tau_{0}} f\left(Y_{s}\right) \mathrm{d} s}\right) .
$$

Fix any $K>1$ and recall from Corollary 17 that there then exists $\mu>0$ such that

$$
f(x) \leqslant K \mathrm{e}^{-\mu x} \quad \forall x \geqslant 0 .
$$

Now fix any $d>0$. Choose a fixed $M \in \mathbb{N}$ sufficiently large such that $K \mathrm{e}^{-\mu y_{1}}<1$ where $y_{1}:=M d$. Then, for any $N \in \mathbb{N}$ and $y_{0}:=(M+N) d$, and with $S_{i}:=\tau_{(M+i-1) d}-\tau_{(M+i) d}$ so that the $S_{i}$ are IID each distributed like the first hitting time of 0 by a Brownian motion started at $d$, we have 


$$
\begin{aligned}
\mathbb{E}_{-\tilde{\rho}}^{y_{0}} \exp \left(\beta \int_{0}^{\tau_{y_{1}}} f\left(Y_{S}\right) \mathrm{d} s\right) & \leqslant \mathbb{E}_{-\tilde{\rho}}^{y_{0}} \exp \left(\beta K \int_{0}^{\tau_{y_{1}}} \mathrm{e}^{-\mu Y_{s}} \mathrm{~d} s\right) \\
& \leqslant \mathbb{E}_{-\tilde{\rho}}^{y_{0}} \exp \left(\beta K \mathrm{e}^{-\mu y_{1}} \sum_{k=1}^{N} \mathrm{e}^{-\mu k d} S_{k}\right) \\
& \leqslant \mathbb{E}_{-\tilde{\rho}}^{y_{0}} \prod_{k=1}^{N} \exp \left(\beta \mathrm{e}^{-\mu k d} S_{k}\right) \\
& =\prod_{k=1}^{N} \mathbb{E}_{-\tilde{\rho}}^{y_{0}} \exp \left(\beta \mathrm{e}^{-\mu k d} S_{k}\right) \\
& =\exp \left(d \sum_{k=1}^{N}\left\{\tilde{\rho}-\sqrt{\tilde{\rho}^{2}-2 \beta \mathrm{e}^{-\mu k d}}\right\}\right) .
\end{aligned}
$$

Since

$$
\tilde{\rho}-\sqrt{\tilde{\rho}^{2}-2 \beta \mathrm{e}^{-\mu k d}}=\left(\frac{\beta}{\sqrt{\rho^{2}+2 \beta}}\right) \mathrm{e}^{-\mu k d}+\mathrm{o}\left(\mathrm{e}^{-\mu k d}\right),
$$

the sum appearing in (21) is convergent when $N \rightarrow \infty$. Using this fact together with monotone convergence and Eqs. (19) and (20) now gives the required result.

\section{Right most particle asymptotic $-\infty<\rho<\sqrt{2 \beta}$}

The intention of Theorem 11 was to establish properties of the probability of extinction in order to justify it as a solution to the travelling-wave equation. However, considering parts (i) and (iii) of this same theorem there is reason to believe that like the $(-\rho, \beta ; \mathbb{R})$-BBM, the $\left(-\rho, \beta ; \mathbb{R}^{+}\right)$-BBM has a right most particle with asymptotic drift $\sqrt{2 \beta}-\rho$ (but now it is necessary to specify that this happens on the survival set). This is indeed the case. After considerable extra work Lemma 2 will actually follow from the stronger result given in Theorem 5, but we can already give a direct alternative proof using intuition from the spine ideas which we include for now for interest.

Proof of Lemma 2. We shall prove this result by establishing separately that

$$
\liminf _{t \uparrow \infty} \frac{R_{t}}{t} \geqslant \sqrt{2 \beta}-\rho \quad \text { and } \quad \limsup _{t \uparrow \infty} \frac{R_{t}}{t} \leqslant \sqrt{2 \beta}-\rho \quad \text { on }\{\zeta=\infty\} P^{x} \text {-a.s. }
$$

Theorem 9 shows that for each $x>0$, on $\{\zeta=\infty\}$, $\lim \sup _{t \uparrow \infty} R_{t}=\infty P^{x}$-almost surely and hence $\sigma_{y}:=$ $\inf \left\{t \geqslant 0: X^{-\rho}(y, \infty)>0\right\}$ is $P^{x}$-almost surely finite for each $y>0$ on $\{\zeta=\infty\}$. This implies that for any $\lambda>0$,

$$
P^{x}\left(\liminf _{t \uparrow \infty} R_{t} / t \geqslant \lambda, \zeta=\infty\right)=P^{x}\left(\liminf _{t \uparrow \infty} R_{t} / t \geqslant \lambda, \sigma_{y}<\infty, \zeta=\infty\right) .
$$

It thus follows that for any $y>x$

$$
\begin{aligned}
P^{x}\left(\liminf _{t \uparrow \infty} R_{t} / t \geqslant \lambda, \zeta=\infty\right) & =P^{x}\left(\sigma_{y}<\infty\right) P^{x}\left(\liminf _{t \uparrow \infty} R_{t} / t \geqslant \lambda, \zeta=\infty \mid \sigma_{y}<\infty\right) \\
& \geqslant P^{x}\left(\sigma_{y}<\infty\right) P^{y}\left(\liminf _{t \uparrow \infty} R_{t} / t \geqslant \lambda, \zeta=\infty\right)
\end{aligned}
$$


where the inequality follows from the fact that at time $\sigma_{y}$ there is one particle positioned at $y$ which, given $\mathcal{F}_{\sigma_{y}}$, gives rise to an branching tree independent of other particles alive at time $\sigma_{y}$ and further whose right most particle is bounded above by the right most particle of $X^{-\rho}$. Recalling Theorem 9, now note that as $y \rightarrow \infty$,

$$
P^{x}\left(\sigma_{y}<\infty\right) \uparrow P^{x}\left(\limsup _{t \uparrow \infty} R_{t}=\infty\right)=P^{x}(\zeta=\infty) .
$$

It follows from (22) with the help of Theorem 11(iii) that when we further insist that $\lambda \in(0, \sqrt{2 \beta}-\rho)$,

$$
\begin{aligned}
P^{x}(\zeta=\infty) & \geqslant P^{x}\left(\liminf _{t \uparrow \infty} R_{t} / t \geqslant \lambda, \zeta=\infty\right) \\
& \geqslant \lim _{y \uparrow \infty} P^{x}\left(\sigma_{y}<\infty\right) P^{y}\left(\liminf _{t \uparrow \infty} R_{t} / t \geqslant \lambda, \zeta=\infty\right) \\
& =P^{x}(\zeta=\infty) .
\end{aligned}
$$

We thus deduce that for any $\varepsilon>0, P^{x}$-almost everywhere on the event $\{\zeta=\infty\}$ we have

$$
\liminf _{t \uparrow \infty} R_{t} / t \geqslant \sqrt{2 \beta}-\rho-\varepsilon .
$$

Additionally note that on $\{\zeta=\infty\}, R_{t}$ is $P^{x}$-almost surely stochastically bounded above by the right-most particle $\mathcal{R}_{t}$ of the unkilled $(-\rho, \beta ; \mathbb{R})$-BBM and recall, for example, $\left.\left.Z_{\lambda}(t) \geqslant \exp \left\{(\lambda+\rho) \mathcal{R}_{t}-\frac{1}{2}\left(\lambda^{2}-\rho^{2}\right) t-\beta t\right)\right\}\right)$, yielding

$$
\limsup _{t \uparrow \infty} R_{t} / t \leqslant \sqrt{2 \beta}-\rho
$$

$P^{x}$-almost everywhere on the event $\{\zeta=\infty\}$.

\section{Proofs of the martingale results}

We first prove the following result, which identifies the speed of particles which contribute to the limit of $W_{\lambda}$.

Theorem 19. For any small $\varepsilon>0$ we have, $P^{x}$-almost-surely,

$$
W_{\lambda}(\infty)=\lim _{t \rightarrow \infty} \sum_{u \in N_{t}^{-\rho}}\left(1-\mathrm{e}^{-2 \lambda Y_{u}(t)}\right) \mathrm{e}^{(\lambda+\rho) Y_{u}(t)-\left(\frac{1}{2}\left(\lambda^{2}-\rho^{2}\right)+\beta\right) t} \mathbf{1}_{\left(\left|Y_{u}(t) / t-\lambda\right|<\varepsilon\right)} .
$$

Proof. Define $E_{\lambda}=E(\lambda):=\frac{1}{2}\left(\lambda^{2}-\rho^{2}\right)+\beta$, let $\varepsilon>0$ be small, and set $\mu:=\lambda-\varepsilon$. Then

$$
\begin{aligned}
& \sum_{u \in N_{t}^{-\rho}}\left(1-\mathrm{e}^{-2 \lambda Y_{u}(t)}\right) \mathrm{e}^{(\lambda+\rho) Y_{u}(t)-E_{\lambda} t} \mathbf{1}_{\left(0<Y_{u}(t) \leqslant(\lambda-\varepsilon) t\right)} \\
& \leqslant \sum_{u \in N_{t}^{-\rho}} \mathrm{e}^{(\mu+\rho) Y_{u}(t)-E_{\mu} t} \mathbf{1}_{\left(0<Y_{u}(t) \leqslant(\lambda-\varepsilon) t\right)} \times \mathrm{e}^{(\lambda-\mu) Y_{u}(t)-\left(E_{\lambda}-E_{\mu}\right) t} \\
& \leqslant \mathrm{e}^{-\frac{1}{2} \varepsilon^{2} t} \sum_{u \in \mathcal{N}_{t}^{-\rho}} \mathrm{e}^{(\mu+\rho) \mathcal{Y}_{u}(t)-E_{\mu} t}=\mathrm{e}^{-\frac{1}{2} \varepsilon^{2} t} Z_{\mu}(t),
\end{aligned}
$$

where $Z_{\mu}(t)$ is the martingale given in Eq. (3), which is positive and hence almost-surely convergent. Hence, $P^{x}$-almost-surely,

$$
\limsup _{t \rightarrow \infty} \sum_{u \in N_{t}^{-\rho}}\left(1-\mathrm{e}^{-2 \lambda Y_{u}(t)}\right) \mathrm{e}^{(\lambda+\rho) Y_{u}(t)-E_{\lambda} t} \mathbf{1}_{\left(0<Y_{u}(t) \leqslant(\lambda-\varepsilon) t\right)}=0 .
$$


The other bound can be proved similarly by setting $\mu:=\lambda+\varepsilon$ and showing

$$
\sum_{u \in N_{t}^{-\rho}}\left(1-\mathrm{e}^{-2 \lambda Y_{u}(t)}\right) \mathrm{e}^{(\lambda+\rho) Y_{u}(t)-E_{\lambda} t} \mathbf{1}_{\left(Y_{u}(t) \geqslant(\lambda+\varepsilon) t\right)} \leqslant \mathrm{e}^{-\frac{1}{2} \varepsilon^{2} t} Z_{\mu}(t)
$$

as required.

Remark 20. It is implicit in the proof of Theorem 19 that we also have another representation for $W_{\lambda}(\infty)$ :

$$
W_{\lambda}(\infty)=\lim _{t \rightarrow \infty} \sum_{u \in N_{t}^{-\rho}} \mathrm{e}^{(\lambda+\rho) Y_{u}(t)-\left(\frac{1}{2}\left(\lambda^{2}-\rho^{2}\right)+\beta\right) t} .
$$

That is, the limit of $W_{\lambda}$ is the same as that part of the limit of $Z_{\lambda}$ contributed from particles that avoided the origin.

Recall that $P^{x}(\zeta=\infty)>0$ if and only if $\rho<\sqrt{2 \beta}$, and note that if this condition does not hold there can be no values of $\lambda$ for which $W_{\lambda}$ is uniformly integrable. When $\rho \geqslant \sqrt{2 \beta}$, the critical $\lambda$ value of $\sqrt{2 \beta}-\rho$ in Theorem 4 corresponds to the right-most particle asymptotic of Lemma 2, tallying with the intuitive notion that the $W_{\lambda}$ martingale limit measures particles travelling at speed $\lambda$.

Proof of Theorem 4. We note that, for $\lambda, x>0$ and for each $p \in(1,2]$ :

1. The martingale $W_{\lambda}$ is $\mathcal{L}^{p}\left(P^{x}\right)$-convergent if $p(\lambda+\rho)^{2} / 2<\beta$;

2. Almost surely under $P^{x}, W_{\lambda}(\infty)=0$ when $(\lambda+\rho)^{2} / 2 \geqslant \beta$.

This $\mathcal{L}^{p}$-convergence result for $W_{\lambda}$ follows from the trivial bound $W_{\lambda} \leqslant Z_{\lambda}$ and known results for $Z_{\lambda}$.

It remains to check that process survival is equivalent to a strictly positive limit for $W_{\lambda}$. From the definition for $W_{\lambda}$, it is clear that $\{\zeta<\infty\} \subseteq\left\{W_{\lambda}(\infty)=0\right\}$, so that $P^{x}\left(W_{\lambda}(\infty)=0 ; \zeta<\infty\right)=P^{x}(\zeta<\infty)$. We can also write $P^{x}\left(W_{\lambda}(\infty)=0\right)$ as

$$
P^{x}\left(W_{\lambda}(\infty)=0\right)=P^{x}\left(W_{\lambda}(\infty)=0 ; \zeta<\infty\right)+P^{x}\left(W_{\lambda}(\infty)=0 ; \zeta=\infty\right)
$$

and the result follows if we can show that $P^{x}\left(W_{\lambda}(\infty)=0\right)=P^{x}(\zeta<\infty)$. Define $g(x):=P^{x}\left(W_{\lambda}(\infty)=0\right)$, and then, by a similar argument to that used in the proof of Theorem 13

$$
g(x)=E^{x}\left(\prod_{u \in N_{t}^{-\rho}} g\left(Y_{u}(t)\right)\right)
$$

and hence $g(x)$ satisfies the ODE in the system (4), and also the boundary condition $\lim _{x \downarrow 0} g(x)=1$. With the representation of Remark 20 in mind, considering the $(-\rho, \beta ; \mathbb{R})$-BBM path-wise we see that $g(x)$ is monotone decreasing in $x$, and so $g(x) \downarrow g(\infty)$ as $x \rightarrow \infty$. Now for any fixed time $t>0$, we have $N_{t}^{-\rho} \uparrow \mathcal{N}_{t}^{-\rho}$ as $x \rightarrow \infty$, and, looking at the process path-wise again, we also have $Y_{u}(t) \uparrow \infty$ as $x \rightarrow \infty$, for all $u \in N_{t}^{-\rho}$. Thus we may take limits on both sides of (24) to obtain $g(\infty)=E^{0}\left(\prod_{u \in \mathcal{N}_{t}^{-\rho}} g(\infty)\right)$, whence $g(\infty)=0$ or 1 . Since $W_{\lambda}$ is uniformly integrable for the values of $\lambda$ under consideration we must have $g(\infty)=0$. Hence $g(x)$ satisfies the ODE and boundary conditions in (4), and uniqueness of the one-sided travelling wave completes the argument.

Proof of Theorem 5. The key idea in the proof is to overestimate the indicator functions in (6) by exponentials and then re-arrange the expression to obtain martingale terms. We emphasise that the use of $W_{\lambda}$, as opposed to $Z_{\lambda}$, in the following arguments is absolutely essential but, otherwise, we closely follow Git et al. [16]. Bounding $N_{t}^{-\rho}(\lambda t, \infty)$ above, we have

$$
N_{t}^{-\rho}(\lambda t, \infty)=\sum_{u \in N_{t}^{-\rho}} \mathbf{1}_{\left(Y_{u}(t)-\lambda t \geqslant 0\right)} \leqslant \sum_{u \in \mathcal{N}_{t}^{-\rho}} \mathrm{e}^{(\lambda+\rho)\left(\mathcal{Y}_{u}(t)-\lambda t\right)}=\mathrm{e}^{\Delta(\lambda) t} Z_{\lambda}(t) .
$$


Now if $\lambda \geqslant \sqrt{2 \beta}-\rho$ then $Z_{\lambda}$ has an almost-sure zero limit and $\Delta(\lambda) \leqslant 0$, whence $\sum_{u \in N_{t}^{-\rho}} \mathbf{1}_{\left(Y_{u}(t) \geqslant \lambda t\right)}=0$, eventually, with probability 1 . On the other hand, if $0<\lambda<\sqrt{2 \beta}-\rho$ then $Z_{\lambda}(\infty)>0$ almost surely and $\Delta(\lambda)>0$ so

$$
\limsup _{t \rightarrow \infty} t^{-1} \ln N_{t}^{-\rho}(\lambda t, \infty) \leqslant \Delta(\lambda), \quad \text { a.s. }
$$

For the reverse inequality, let $\varepsilon>0$ be small and $0<\lambda<\sqrt{2 \beta}-\rho$. Then

$$
\sum_{u \in N_{t}^{-\rho}}\left(1-\mathrm{e}^{-2 \lambda Y_{u}(t)}\right) \mathrm{e}^{(\lambda+\rho) Y_{u}(t)-E_{\lambda} t} \mathbf{1}_{\left((\lambda-\varepsilon) t<Y_{u}(t) \leqslant(\lambda+\varepsilon) t\right)} \leqslant \mathrm{e}^{\left((\lambda+\rho) \lambda-E_{\lambda}\right) t} \mathrm{e}^{\varepsilon(\lambda+\rho) t} \sum_{u \in N_{t}^{-\rho}} \mathbf{1}_{\left((\lambda-\varepsilon) t<Y_{u}(t)\right)} .
$$

Noting that $(\lambda+\rho) \lambda-E_{\lambda}=-\Delta(\lambda)$, we obtain

$$
\begin{aligned}
& t^{-1} \ln \sum_{u \in N_{t}^{-\rho}}\left(1-\mathrm{e}^{-2 \lambda Y_{u}(t)}\right) \mathrm{e}^{(\lambda+\rho) Y_{u}(t)-E_{\lambda} t} \mathbf{1}_{\left((\lambda-\varepsilon) t<Y_{u}(t) \leqslant(\lambda+\varepsilon) t\right)} \\
& \leqslant-\Delta(\lambda)+\varepsilon(\lambda+\rho)+t^{-1} \ln \sum_{u \in N_{t}^{-\rho}} \mathbf{1}_{\left((\lambda-\varepsilon) t<Y_{u}(t)\right)} .
\end{aligned}
$$

Now as $t \rightarrow \infty$, it follows from the crucial facts that $W_{\lambda}(\infty)>0$ (from Theorem 4) and that the limit only 'sees' particles of speed $\lambda$ (from Proposition 19) that, on $\{\zeta=\infty\}$, the left-hand side of (25) tends to zero, and since $\varepsilon>0$ is arbitrary, we find that

$$
\liminf _{t \rightarrow \infty} t^{-1} \ln \sum_{u \in N_{t}^{-\rho}} \mathbf{1}_{\left(Y_{u}(t) \geqslant \lambda t\right)} \geqslant \Delta(\lambda), \quad \text { a.s. }
$$

which completes the proof.

\section{Proof of Theorem 6}

The proof of Theorem 6 rests on the close links between branching diffusions and partial differential equations; essentially, the assertion of Theorem 6 is that for large times $t$ the solution of the non-linear equation

$$
\frac{\partial u}{\partial t}=\frac{1}{2} \frac{\partial^{2} u}{\partial x^{2}}-\rho \frac{\partial u}{\partial x}+\beta u(1-u)
$$

with $u \in C^{1,2}\left(\mathbb{R}^{+} \times \mathbb{R}^{+}\right)$and $u(\cdot, 0+)=0$, is asymptotically equal to a constant multiple of the solution of the linearised equation

$$
\frac{\partial w}{\partial t}=\frac{1}{2} \frac{\partial^{2} w}{\partial x^{2}}-\rho \frac{\partial w}{\partial x}+\beta w,
$$

for $w \in C^{1,2}\left(\mathbb{R}^{+} \times \mathbb{R}^{+}\right)$and $w(\cdot, 0+)=0$. Considering $x, y, t>0$, it is classical theory that $u(t, x, y):=$ $P^{x}\left(R_{t}>y\right)$ solves (26) with initial condition $u(0, x, y)=\mathbf{1}_{(x>y)}$. This link can be seen from the product martingales in McKean's work [31,32]. A simple Feynman-Kac argument yields the following:

Proposition 21. Let $w(t, x, y)$ be the solution of the linearised equation (27) with the same initial condition, that is $w(0, x, y)=\mathbf{1}_{(x>y)}$. Then $u(t, x, y) \leqslant w(t, x, y)$ for all $t, x, y>0$.

Remark 22. The probabilistic interpretation of Proposition 21 is that, for all $t, x>0$,

$$
P^{x}\left(R_{t}>\lambda t+\theta\right) \leqslant E^{x}\left(N_{t}^{-\rho}(\lambda t+\theta, \infty)\right) .
$$


Proof of Theorem 6. Let $\left\{\mathbb{B}_{x_{1}, x_{2}}^{t}(\phi): s \in[0, t]\right\}$ be a Brownian bridge that travels from point $x_{1}$ to $x_{2}$ over time period $[0, t]$, and let $\tau_{0}^{t}$ to be the first hitting of the origin by the bridge. Then we have

$$
\begin{aligned}
u(t, x, y) & =\mathbb{E}_{-\rho}^{x}\left\{\mathbf{1}_{\left(Y_{t}>y\right)} \mathrm{e}^{\beta \int_{0}^{t}\left(1-u\left(t-\phi, Y_{\phi}, y\right)\right) \mathrm{d} \phi} ; \tau_{0}>t\right\} \\
& =\mathrm{e}^{\beta t} \int_{0}^{\infty} \mathbb{P}_{-\rho}^{x}\left(Y_{t}-y \in \mathrm{d} z\right) \mathbb{E}\left\{\mathrm{e}^{-\beta \int_{0}^{t} u\left(t-\phi, \mathbb{B}_{x, z+y}^{t}(\phi), y\right) \mathrm{d} \phi} ; \tau_{0}^{t}>t\right\} .
\end{aligned}
$$

Hence

$$
\begin{aligned}
u(t, x, \lambda t+\theta)= & \frac{\mathrm{e}^{-\left(\frac{1}{2}(\lambda+\rho)^{2}-\beta\right) t}}{\sqrt{2 \pi t}} \mathrm{e}^{(\lambda+\rho)(x-\theta)} \int_{0}^{\infty} \mathrm{e}^{-(\lambda+\rho) z} \exp \left(\frac{-(x-\theta-z)^{2}}{2 t}\right) \\
& \times \mathbb{E}\left\{\mathrm{e}^{-\beta \int_{0}^{t} u\left(t-\phi, \mathbb{B}_{x, z+\lambda t+\theta}^{t}(\phi), \lambda t+\theta\right) \mathrm{d} \phi} ; \tau_{0}^{t}>t\right\} \mathrm{d} z,
\end{aligned}
$$

and then, by dominated convergence, to complete the proof of (7) it suffices to show that there exists some function $g:(0, \infty) \rightarrow(0,1]$ such that

$$
\mathbb{E}\left\{\mathrm{e}^{-\beta \int_{0}^{t} u\left(t-\phi, \mathbb{B}_{x, z+\lambda t+\theta}^{t}(\phi), \lambda t+\theta\right) \mathrm{d} \phi} ; \tau_{0}^{t}>t\right\} \underset{t \rightarrow \infty}{\longrightarrow} g(z)\left(1-\mathrm{e}^{-2 \lambda x}\right),
$$

since this would ensure that

$$
\int_{0}^{\infty} \mathrm{e}^{-(\lambda+\rho) z} \exp \left(\frac{-(x-\theta-z)^{2}}{2 t}\right) \mathbb{E}\left\{\mathrm{e}^{-\beta \int_{0}^{t} u\left(t-\phi, \mathbb{B}_{x, z+\lambda t+\theta}^{t}(\phi), \lambda t+\theta\right) \mathrm{d} \phi} ; \tau_{0}^{t}>t\right\} \mathrm{d} z \underset{t \rightarrow \infty}{\longrightarrow} C\left(1-\mathrm{e}^{-2 \lambda x}\right) .
$$

Although we are essentially following the strategy of Chauvin and Rouault [9] here, extra effort is required to deal with the complications arising from the introduction of the absorbing barrier. In particular, Lemma 23 below uses a similar argument to show that a certain expectation converges, but additional work is needed to identify the limit; after this, a careful chosen construction of the family of Brownian bridges from two independent Brownian motions allows us to give intuitive proofs of the remaining new results that yield Theorem 6 .

Let $B:=\{B(s): s \geqslant 0\}$ be a standard Brownian motion started at the origin, then recall the fact that a Brownian bridge $\left\{\mathbb{B}_{x_{1}, x_{2}}^{t}(\phi): \phi \in[0, t]\right\}$ from positions $x_{1}$ to $x_{2}$ can be constructed by taking

$$
\mathbb{B}_{x_{1}, x_{2}}^{t}(\phi)=B(\phi)-\frac{\phi}{t} B(t)+x_{1}+\frac{\phi}{t}\left(x_{2}-x_{1}\right) \quad(0 \leqslant \phi \leqslant t) .
$$

Also define

$$
\tilde{u}(t, x, y):=P^{x}\left(\mathcal{R}_{t}>y\right),
$$

where $\mathcal{R}_{t}$ is the right-most particle in the unkilled $(-\rho, \beta ; \mathbb{R})$-BBM. Note that $\tilde{u}(t, x, y)$ is a solution of (26) as a function of $(t, x) \in \mathbb{R}^{+} \times \mathbb{R}$ with the same initial condition as $u$, that is $\tilde{u}(0, x, y)=\mathbf{1}_{(x>y)}$. We find:

Lemma 23. As $t \rightarrow \infty$,

$$
\mathbb{E}\left(\mathrm{e}^{-\beta \int_{0}^{t} u\left(t-\phi, \mathbb{B}_{x, z+\lambda t+\theta}^{t}(\phi), \lambda t+\theta\right) \mathrm{d} \phi}\right) \rightarrow \mathbb{E}\left(\mathrm{e}^{-\beta \int_{0}^{\infty} \tilde{u}(\phi, B(\phi)+z-\lambda \phi, 0) \mathrm{d} \phi}\right)
$$

where the limit is independent of $\theta$ and $x$.

Proof of Lemma 23. The proof requires only a slight modification of the argument in Chauvin and Rouault [9, Lemma 3.1]. Since the effect of the killing vanishes as the particle's start position tends to infinity, we can show that

$$
\mathbf{1}_{[0, t]}(\phi) u\left(\phi, \mathbb{B}_{z+\lambda t+\theta, x}^{t}(\phi), \lambda t+\theta\right) \underset{t \rightarrow \infty}{\longrightarrow} \tilde{u}(\phi, B(\phi)+z-\lambda \phi, 0) .
$$

Then a dominated-convergence argument shows that the right-hand side of (28) is strictly positive. 
Construction of the bridges. We make the following simultaneous construction of all the Brownian bridges $\mathbb{B}_{x, z}^{t}(\cdot)$ with indices $t>0, x, z>0$ using two independent Brownian motions started at the origin, $W=\{W(s)$ : $s>0\}$ and $X=\{X(s): s>0\}$ :

$$
\mathbb{B}_{x, z}^{t}(\phi):= \begin{cases}W(\phi)-\frac{\phi}{t} W(t)+x+\frac{\phi}{t}(z-x), & \text { for } \phi \in\left[0, \tau_{0}^{t}\right), \\ \widetilde{\mathbb{B}}_{z, 0}^{t-\tau_{0}^{t}(t-\phi),} & \text { for } \phi \in\left[\tau_{0}^{t}, t\right],\end{cases}
$$

where

$$
\tau_{0}^{t}=\tau_{0}^{t}(x, z)=\inf \left\{\phi \geqslant 0: W(\phi)-\frac{\phi}{t} W(t)+x+\frac{\phi}{t}(z-x)=0\right\}
$$

and for any $s>0$

$$
\widetilde{\mathbb{B}}_{z, 0}^{s}(u):=X(u)-\frac{u}{s} X(s)+z-\frac{u}{s} z \quad \text { for } u \in[0, s] .
$$

Note that $\tau_{0}^{t}$ is determined entirely from the path of $W$, and, almost surely as $t \rightarrow \infty$, we have

$$
\tau_{0}^{t}(x, z+\lambda t+\theta) \rightarrow \tau_{0}:=\inf \{\phi: W(\phi)+\lambda \phi+x=0\} .
$$

Also

$$
\mathbb{B}_{x, z+\lambda t+\theta}^{t}(\phi) \rightarrow W(\phi)+\lambda \phi+x
$$

uniformly on any interval $[0, s]$ where $s<\tau_{0}$.

Lemma 24. On the event $\left\{\tau_{0}<\infty\right\}$,

$$
\int_{0}^{\tau_{0}^{t}} u\left(t-\phi, \mathbb{B}_{x, z+\lambda t+\theta}^{t}(\phi), \lambda t+\theta\right) \mathrm{d} \phi \rightarrow 0
$$

almost surely as $t \rightarrow \infty$.

Proof of Lemma 24. Let $s<\tau_{0}<\infty$. Then since $\tau_{0}^{t} \rightarrow \tau_{0}$ almost surely, there exists some $t_{0}$ such that $s<\tau_{0}^{t}$ for all $t \geqslant t_{0}$. Recalling the function $w$ from Proposition 21, we note that

$$
\begin{aligned}
w(t, x, y) & =\mathrm{e}^{\beta t} \mathbb{E}_{-\rho}^{x}\left(\mathbf{1}_{\left(Y_{t}>y\right)} ; \tau_{0}>t\right) \\
& \leqslant \mathrm{e}^{\beta t} \mathbb{E}_{0}^{0}\left(\mathbf{1}_{\left(Y_{t}>y-x+\rho t\right)}\right)=: \widetilde{w}(t, y-x),
\end{aligned}
$$

and Chernov's inequality gives

$$
\widetilde{w}(s, z) \leqslant \exp \left(\beta s-\frac{z^{2}}{2 s}\right), \quad \forall s, z>0 .
$$

Using Proposition 21 and the inequalities (32) and (33) we have, for any $B \in \mathbb{R}$,

$$
u(t-\phi, B, \lambda t+\theta) \leqslant \mathrm{e}^{\left(\beta-\frac{1}{2}(\lambda+\rho)^{2}\right) t} \times \mathrm{e}^{-\beta \phi-\frac{1}{2}(\lambda+\rho)^{2} \phi+(B-\theta)(\lambda+\rho)} .
$$

Then, combining these facts with (31), we see that 


$$
\begin{aligned}
\mathrm{e}^{\left(\frac{1}{2}(\lambda+\rho)-^{2} \beta\right) t} \int_{0}^{s} u\left(t-\phi, \mathbb{B}_{x, z+\lambda t+\theta}^{t}(\phi), \lambda t+\theta\right) \mathrm{d} \phi & \leqslant \int_{0}^{s} \mathrm{e}^{-\beta \phi-\frac{1}{2}(\lambda+\rho)^{2} \phi+\left(\mathbb{B}_{x, z+\lambda t+\theta}^{t}(\phi)-\theta\right)(\lambda+\rho)} \mathrm{d} \phi \\
& \rightarrow \int_{0}^{s} \mathrm{e}^{-\beta \phi-\frac{1}{2}(\lambda+\rho)^{2} \phi+(W(\phi)+\lambda \phi+x-\theta)(\lambda+\rho)} \mathrm{d} \phi<\infty
\end{aligned}
$$

as $t \rightarrow \infty$. Since $\lambda>\sqrt{2 \beta}-\rho$ and the above holds for all $s<\tau_{0}<\infty$, the lemma follows.

Define

$$
I(z):=\int_{0}^{\infty} \tilde{u}(\phi, X(\phi)+z-\lambda \phi, 0) \mathrm{d} \phi
$$

and note that this definition is independent of $W$ (hence also of each $\tau_{0}^{t}$ and $\tau_{0}$ ).

Lemma 25. On the event $\left\{\tau_{0}<\infty\right\}$,

$$
\int_{0}^{t} u\left(t-\phi, \mathbb{B}_{x, z+\lambda t+\theta}^{t}(\phi), \lambda t+\theta\right) \mathrm{d} \phi \rightarrow I(z),
$$

almost surely as $t \rightarrow \infty$. In particular, note limit $I(z) \in(0, \infty)$ is independent of $\theta$ and $x$.

Proof. Using the construction at (29), we see that

$$
\begin{aligned}
\int_{\tau_{0}^{t}}^{t} u\left(t-\phi, \mathbb{B}_{x, z+\lambda t+\theta}^{t}(\phi), \lambda t+\theta\right) \mathrm{d} \phi & =\int_{\tau_{0}^{t}}^{t} u\left(t-\phi, \widetilde{\mathbb{B}}_{z+\lambda t+\theta, 0}^{t-\tau_{0}^{t}}(t-\phi), \lambda t+\theta\right) \mathrm{d} \phi \\
& =\int_{0}^{t-\tau_{0}^{t}} u\left(\phi, \widetilde{\mathbb{B}}_{z+\lambda t+\theta, 0}^{t-\tau_{0}^{t}}(\phi), \lambda t+\theta\right) \mathrm{d} \phi .
\end{aligned}
$$

We now note that, because of our construction of $\widetilde{\mathbb{B}}_{z+\lambda t+\theta, 0}^{t-\tau_{0}^{t}}$ at (30), we may almost exactly mirror the argument required for Lemma 23 to show that (34) converges to $I(z)$ as $t \rightarrow \infty$.

Immediately from Lemma 25, we see that

$$
\mathbb{E}\left\{\mathrm{e}^{-\beta \int_{0}^{t} u\left(t-\phi, \mathbb{B}_{x, z+\lambda t+\theta}^{t}(\phi), \lambda t+\theta\right) \mathrm{d} \phi} ; \tau_{0}^{t}<t\right\} \underset{t \rightarrow \infty}{\longrightarrow} \mathbb{E}\left(\mathrm{e}^{-\beta I(z)} ; \tau_{0}<\infty\right) .
$$

To complete the proof of (7), we note that $I(z)$ and $\tau_{0}$ are independent by construction, $\mathbb{P}^{x}\left(\tau_{0}<\infty\right)=\mathrm{e}^{-2 \lambda x}$ and the right-hand side of Eq. (28) is the same as $\mathbb{E}\left(\mathrm{e}^{-I(z)}\right)$, hence

$$
\mathbb{E}\left\{\mathrm{e}^{-\beta \int_{0}^{t} u\left(t-\phi, \mathbb{B}_{x, z+\lambda t+\theta}^{t}(\phi), \lambda t+\theta\right) \mathrm{d} \phi} ; \tau_{0}^{t}>t\right\} \underset{t \rightarrow \infty}{\longrightarrow}\left(1-\mathrm{e}^{-2 \lambda x}\right) \mathbb{E}\left(\mathrm{e}^{-\beta I(z)}\right)
$$

as required. Finally, we note that the equivalent statement of Eq. (8) can be deduced from Eq. (7), the many-to-one lemma (9), and the one-particle calculation

$$
\mathbb{P}_{-\rho}^{x}\left(Y(t) \geqslant \lambda t+\theta ; \tau_{0}>t\right) \underset{t \rightarrow \infty}{\sim} \frac{1}{(\lambda+\rho) \sqrt{2 \pi t}}\left(1-\mathrm{e}^{-2 \lambda x}\right) \mathrm{e}^{(\lambda+\rho)(x-\theta)-\frac{1}{2}(\lambda+\rho)^{2} t} .
$$

This completes the proof of Theorem 6. 


\section{Acknowledgements}

We thank the referee for their careful reading of the manuscript, and for some very valuable comments which lead to a considerably improved version of the original manuscript. This work was conducted whilst J.W. Harris was in receipt of an EPSRC research studentship. S.C. Harris and A.E. Kyprianou are pleased to acknowledge the support of the joint NWO-British Council Anglo-Dutch research grant Nr. BR 61-461.

\section{References}

[1] D.G. Aronson, H.F. Weinberger, Nonlinear diffusions in population genetics, combustion and nerve propagation, in: J. Goldstein (Ed.), Partial Differential Equations and Related Topics, in: Lecture Notes in Math., vol. 446, Springer-Verlag, Berlin, 1975.

[2] K.B. Athreya, Change of measures for Markov chains and the $L \log L$ theorem for branching processes, Bernoulli 6 (2) (2000) $323-338$.

[3] J.D. Biggins, A.E. Kyprianou, Measure change in multitype branching, Adv. Appl. Probab. 36 (2) (2004) 544-581.

[4] A.N. Borodin, P. Salminen, Handbook of Brownian Motion-Facts and Formulae, Probability and its Applications, Birkhäuser, Basel, 1996.

[5] M.D. Bramson, Maximal displacement of branching Brownian motion, Comm. Pure Appl. Math. 31 (5) (1978) 531-581.

[6] M.D. Bramson, Convergence of solutions of the Kolmogorov equation to travelling waves, Mem. Amer. Math. Soc. 44 (285) (1983), iv +190 .

[7] A. Champneys, S.C. Harris, J. Toland, J. Warren, D. Williams, Algebra, analysis and probability for a coupled system of reaction-diffusion equations, Philos. Trans. Roy. Soc. London 350 (1995) 69-112.

[8] B. Chauvin, Product martingales and stopping lines for branching Brownian motion, Ann. Probab. 19 (3) (1991) $1195-1205$.

[9] B. Chauvin, A. Rouault, KPP equation and supercritical branching Brownian motion in the subcritical speed area. Application to spatial trees, Probab. Theory Related Fields 80 (2) (1988) 299-314.

[10] B. Chauvin, A. Rouault, Supercritical branching Brownian motion and K-P-P equation in the critical speed-area, Math. Nachr. 149 (1990) $41-59$.

[11] E.A. Coddington, N. Levinson, Theory of Ordinary Differential Equations, McGraw-Hill Book Company, New York, 1955.

[12] E.B. Dynkin, Superprocesses and partial differential equations, Ann. Probab. 21 (3) (1993) 1185-1262.

[13] J. Engländer, A.E. Kyprianou, Local extinction versus local exponential growth for spatial branching processes, Ann. Probab. 32 (1A) (2004) 78-99.

[14] R.A. Fisher, The advance of advantageous genes, Ann. Eugenics 7 (1937) 355-369.

[15] M. Freidlin, Functional Integration and Partial Differential Equations, Ann. Math. Stud., vol. 109, Princeton University Press, Princeton, NJ, 1985.

[16] Y. Git, J.W. Harris, S.C. Harris, Exponential growth-rates and large deviations for a typed branching diffusion, University of Bath preprint, 2004.

[17] R. Hardy, S.C. Harris, A new formulation of the spine approach, University of Bath preprint, 2004.

[18] S.C. Harris, Travelling-waves for the FKPP equation via probabilistic arguments, Proc. Roy. Soc. Edinburgh Sect. A 129 (3) (1999) 503-517.

[19] S.C. Harris, D. Williams, Large deviations and martingales for a typed branching diffusion. I, Astérisque 236 (1996) 133-154. Hommage à P.A. Meyer et J. Neveu.

[20] N. Ikeda, M. Nagasawa, S. Watanabe, Branching Markov processes. I, J. Math. Kyoto Univ. 8 (1968) 233-278.

[21] N. Ikeda, M. Nagasawa, S. Watanabe, Branching Markov processes. II, J. Math. Kyoto Univ. 8 (1968) 365-410.

[22] N. Ikeda, M. Nagasawa, S. Watanabe, Branching Markov processes. III, J. Math. Kyoto Univ. 9 (1969) 95-160.

[23] Y. Kametaka, On the nonlinear diffusion equation of Kolmogorov-Petrovskii-Piskunov type, Osaka J. Math. 13 (1) (1976) 11-66.

[24] H. Kesten, Branching Brownian motion with absorption, Stochastic Process. Appl. 7 (1) (1978) 9-47.

[25] A.N. Kolmogorov, I. Petrovski, N. Piscounov, Étude de l'équation de la diffusion avec croissance de la quantité de matière et son application à un problem biologique, Mosc. Univ. Bull. Math. 1 (1937) 1-25. Translated and reprinted in P. Pelce Dynamics of Curved Fronts, Academic, San Diego, 1988.

[26] D. Kuhlbusch, On weighted branching processes in random environment, Stochastic Process. Appl. 109 (1) (2004) $113-144$.

[27] A.E. Kyprianou, Travelling wave solutions to the K-P-P equation: alternatives to Simon Harris' probabilistic analysis, Ann. Inst. H. Poincaré Probab. Statist. 40 (1) (2004) 53-72.

[28] A.E. Kyprianou, A. Rahimzadeh Sani, Martingale convergence and the functional equation in the multi-type branching random walk, Bernoulli 7 (4) (2001) 593-604.

[29] R. Lyons, R. Pemantle, Y. Peres, Conceptual proofs of $L \log L$ criteria for mean behavior of branching processes, Ann. Probab. 23 (3) (1995) 1125-1138. 
[30] R. Lyons, A simple path to Biggins' martingale convergence for branching random walk, in: K.B. Athreya, P. Jagers (Eds.), Classical and Modern Branching Processes, Minneapolis, MN, 1994, in: IMA Vol. Math. Appl., vol. 84, Springer, New York, 1997, pp. $217-221$.

[31] H.P. McKean, Application of Brownian motion to the equation of Kolmogorov-Petrovskiir-Piskunov, Comm. Pure Appl. Math. 28 (3) (1975) 323-331.

[32] H.P. McKean, A correction to: "Application of Brownian motion to the equation of Kolmogorov-Petrovskiı̌-Piskunov" (Comm. Pure Appl. Math. 28 (3) (1975) 323-331), Comm. Pure Appl. Math. 29 (5) (1976) 553-554.

[33] J. Neveu, Multiplicative martingales for spatial branching processes, in: Seminar on Stochastic Processes, Princeton, NJ, 1987, in: Progr. Probab. Statist., vol. 15, Birkhäuser Boston, Boston, MA, 1988, pp. 223-242.

[34] P. Olofsson, The $x \log x$ condition for general branching processes, J. Appl. Probab. 35 (3) (1998) 537-544.

[35] R.G. Pinsky, K-P-P-type asymptotics for nonlinear diffusion in a large ball with infinite boundary data and on $\mathbf{R}^{d}$ with infinite initial data outside a large ball, Comm. Partial Differential Equations 20 (7-8) (1995) 1369-1393.

[36] K. Uchiyama, The behavior of solutions of some nonlinear diffusion equations for large time, J. Math. Kyoto Univ. 18 (3) (1978) $453-508$.

[37] S. Watanabe, On the branching process for Brownian particles with an absorbing boundary, J. Math. Kyoto Univ. 4 (1965) 385-398. 